\title{
Dynamics of transposable elements generates structure and symmetries in genetic sequences
}

\author{
Giampaolo Cristadoro $\odot,{ }^{1, *}$ Mirko Degli Esposti $\odot,{ }^{2}$ and Eduardo G. Altmann $\odot^{3}$ \\ ${ }^{1}$ Dipartimento di Matematica e Applicazioni, Università di Milano - Bicocca, Via Roberto Cozzi 55, I-20125 Milan, Italy \\ ${ }^{2}$ Department of Computer Science and Engineering, University of Bologna, Mura Anteo Zamboni 7, 40126 Bologna, Italy \\ ${ }^{3}$ School of Mathematics and Statistics F07, University of Sydney NSW 2006, Australia
}

(Received 26 August 2019; accepted 18 August 2020; published 23 September 2020)

\begin{abstract}
Genetic sequences are known to possess nontrivial composition together with symmetries in the frequencies of their components. Recently, it has been shown that symmetry and structure are hierarchically intertwined in DNA, suggesting a common origin for both features. However, the mechanism leading to this relationship is unknown. Here we investigate a biologically motivated dynamics for the evolution of genetic sequences. We show that a metastable (long-lived) regime emerges in which sequences have symmetry and structure interlaced in a way that matches that of extant genomes.
\end{abstract}

DOI: 10.1103/PhysRevResearch.2.033477

Introduction. Transposable elements (TEs) are DNA sequences that can relocate themselves in new sites of the genome. They were first discovered in maize by McClintock in the mid-1940s and initially considered as parasites with no functional roles [1]. Nowadays TEs are known to be ubiquitous in both prokaryotes and eukaryotes genomes [2,3] and little doubts are left of their prominent role in genome evolution, shaping structure and function in a multitude of ways [4,5]. As TEs constitute more than half of the sequence in many higher eukaryotes, a fingerprint of their presence can be quantitatively extracted from the statistical properties of their host DNA. Indeed, TEs properties were shown to be crucial in explaining structural global features of genome sequences [6-11].

Recently, Albrecht-Buehler [12] suggested that TEs were the main driving force for the emergence of the second Chargaff parity rule. This rule states that, in each strand of the DNA, the frequency of a short oligonucleotide $\mathbf{w}$ is approximately equal to that of its symmetrically related $\hat{\mathbf{w}}$, obtained from $\mathbf{w}$ by reversing the order of the symbols and substituting each nucleotide with its conjugated $A \leftrightarrow T$ and $C \leftrightarrow G$ (e.g., $\mathbf{w}=A C T G G C T, \hat{\mathbf{w}}=A G C C A G T)$ ). It has been first observed by Chargaff in the 1950s [13] and since then detected across different organisms leading to different proposals for its origin and function [14-29]. The importance of the Albrecht-Buehler explanation is that it shows how this symmetry naturally emerges as an asymptotic outcome of the cumulative action of inversions/transpositions, one of the main mechanisms of relocation of TEs. As we will show, while the proposed mechanism nicely induces Chargaff symmetry in the asymptotic DNA, it does it at

\footnotetext{
*Corresponding author: giampaolo.cristadoro@unimib.it
}

Published by the American Physical Society under the terms of the Creative Commons Attribution 4.0 International license. Further distribution of this work must maintain attribution to the author(s) and the published article's title, journal citation, and DOI. the cost of trivialization of the structural properties of the sequence: Symmetry is obtained because of the complete randomization of the full double-stranded DNA. In view of the ubiquity of complex structures in genomes [30-38], this result raises the question whether symmetry can appear without a full randomization of the sequence and in a way that is compatible with the existence of structure. The importance of this question is enhanced by our recent findings [39] that Chargaff symmetry extends beyond the frequencies of short oligonucleotides-remaining valid on scales where nontrivial structure is present-and that a hierarchy of other symmetries exists, nested at different structural scales. This findings are confirmed in Fig. 1, which shows how commonly used indicators of structures, such as recurrence-time distribution [Fig. 1(a)] and correlation functions [Fig. 1(b)], coincide for symmetrically related observables at different scales.

In this paper we present a biologically motivated dynamical process that explains the observed relation between symmetry and structure in DNA sequences. In particular, we propose a model that mimics the action (inversions/transpositions) of TEs on DNA and we analytically describe its dynamical behavior. Using indicators to quantify both symmetry and the presence of nontrivial structure in symbolic sequences, we show that the co-occurrence of symmetry and structure is an emergent statistical property in sequences generated by such a model, reproducing the same hierarchical relation detected in extant genomes.

Quantifying structure and symmetry. We consider symbolic sequences $\mathbf{s}=\left\{s_{i}\right\}_{i=1}^{N}$ of length $|\mathbf{s}|=N$ with $s_{i} \in \mathcal{A}=$ $\{A, C, G, T\}$. Given a subsequence $\mathbf{a}$ of $\mathbf{s}$ (a word) we denote its corresponding reverse-complemented word as $\hat{\mathbf{a}}$, obtained from a by reversing the order of the symbols and substituting each nucleotide $\{A, C, G, T\}$ by its complementary one $A \leftrightarrow T$ and $C \leftrightarrow G$. We call $f_{x}(\mathbf{s})$ the percentage of the nucleotide $x$ in the sequence $\mathbf{s}$. Finally, we denote by $C G(\mathbf{s}):=$ $f_{C}(\mathbf{s})+f_{G}(\mathbf{s})$ (the so called CG content). In the following, it will be useful to partition the full set $\mathcal{A}^{N}$ into disjoint subsets of fixed $\mathrm{CG}$ content $\mathcal{B}^{N}(k):=\left\{\mathbf{s} \in \mathcal{A}^{N} \mid C G(\mathbf{s})=k / N\right\}$; $\mathcal{A}^{N}=\cup_{k=0}^{N} \mathcal{B}^{N}(k)$. 

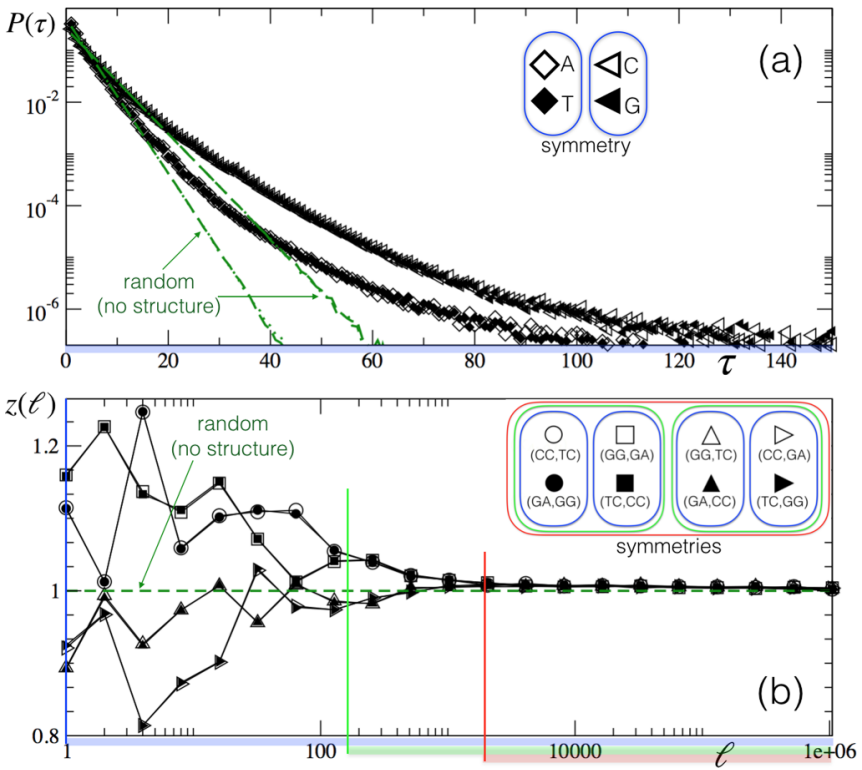

FIG. 1. Symmetry and structure are intertwined in DNA. Results are shown for Homo sapiens chromosome 1 (symbols) and its randomly shuffled version (dashed lines). Each curve corresponds to one observable. Symmetrically related observables appear in the same box in the legend. (a) Distribution $P(\tau)$ of recurrence times $\tau$ (measured in number of basis) between successive occurrences of the same nucleotide. (b) Probability $f_{X_{A}, X_{B}}(\ell)$ that the bigrams $X_{A}$ and $X_{B}$ appear separated by a distance $\ell$. Plotted is the normalized cross-correlation $z_{\left[X_{A}, X_{B}\right]}(\ell)=f_{X_{A}, X_{B}} /\left(f_{X_{A}} f_{X_{B}}\right)$ as a function of $\ell$, for symmetrically related couples $\left[X_{A}, X_{B}\right]$ (see legend). Different nested symmetries are valid at different scales $\ell$ (see Ref. [39] for further details): for $\ell \lesssim 150$ Chargaff $z_{\left[X_{A}, X_{B}\right]}=z_{\left[\hat{X}_{B}, \hat{X}_{A}\right]}$, for $150 \lesssim \ell \lesssim 1500$ Chargaff and reverse symmetry $z_{\left[X_{A}, X_{B}\right]}=z_{\left[X_{B}, X_{A}\right]}$, and for $\ell \gtrsim 1500$ complement $z_{\left[X_{A}, X_{B}\right]}=z_{\left[\hat{X}_{A}, \hat{X}_{B}\right]}$ and reverse symmetry.

We introduce the following simple indicators of the presence of Chargaff symmetry and of nontrivial structure composition of a given sequence $\mathbf{s}$.

To quantify the compliance of $\mathbf{s}$ with Chargaff symmetry, we average the normalized difference of the abundance between a nucleotide and its symmetric one (see [21] where a similar measure was first introduced)

$$
I_{\text {sym }}(\mathbf{s})=\frac{1}{4} \sum_{x \in \mathcal{A}} \frac{\left|f_{x}(\mathbf{s})-f_{\hat{x}}(\mathbf{s})\right|}{f_{x}(\mathbf{s})+f_{\hat{x}}(\mathbf{s})} .
$$

$I_{\text {sym }}=0$ indicates a fully Chargaff-symmetric sequence, $I_{\text {sym }}=1$ is obtained for a sequence for which Chargaff is perfectly violated $\left(f_{A}=f_{C}=0.5, f_{T}=f_{G}=0\right)$, and $I_{\text {sym }}=$ 0.08 is obtained for a $2 \%$ variation of equal frequencies (e.g., $f_{A}=f_{C}=0.23, f_{T}=f_{G}=0.27$ ). For simplicity, we consider $I_{\text {sym }}>0.08$ to be a violation of Chargaff symmetry.

To quantify the presence of nontrivial structures in a given symbolic sequence $\mathbf{s}$ we first compute the distribution $P(\tau)$ of distances $\tau$ between two successive occurrences of the same nucleotide $x$. For random sequences, $P(\tau)$ decays exponentially as $P(\tau)=f_{x}\left(1-f_{x}\right)^{\tau-1}$ and thus has average $1 / f_{x}$ and standard deviation $\sqrt{1-f_{x}} / f_{x}$ (which is $\approx 1 / f_{x}$ for small $f_{x}$ ). In contrast, the presence of a fat tail (standard deviation much larger than the mean) is considered a signature of a complex organization. We thus quantify structure as the distance of $\mathbf{s}$ from random sequences by

$$
I_{\text {str }}(\mathbf{s})=\frac{1}{4} \sum_{x \in \mathcal{A}}\left(\frac{1}{\sqrt{1-f_{x}(\mathbf{s})}} \frac{\sigma_{\tau}(x)}{\mu_{\tau}(x)}-1\right),
$$

where $\mu_{\tau} \equiv\langle\tau\rangle$ and $\sigma_{\tau} \equiv \sqrt{\left\langle\tau^{2}\right\rangle-\langle\tau\rangle^{2}}$ are the mean and standard deviation of the measured $P(\tau)$, and $\sqrt{1-f_{x}}$ is the expected $\sigma_{\tau} / \mu_{\tau}$ for nucleotide $x$ in a random sequence. For random sequence we thus have $I_{\text {str }}(\mathbf{s})=0$, while departure from this value mark the presence of nontrivial structure. For simplicity, we consider $I_{\text {str }}>0.01$ to be a signature of structure.

Dynamics. We investigate symmetry and structure of sequences that evolve through the following dynamics, that maps one sequence $\mathbf{s}(t) \in \mathcal{A}^{N}$ into another sequence $\mathbf{s}(t+1) \in \mathcal{A}^{N}$ by mimicking the action of TEs [12]. The dynamics is defined composing two actions:

(i) Pick a random position $j$ of $\mathbf{s}$ and a random size $\ell \geqslant 0$, with $\langle\ell\rangle=L[51]$.

(ii) Replace the subsequence $\mathbf{b} \equiv\left\{s_{i}\right\}_{i=j}^{j+\ell-1}$ of size $\ell$ starting at position $j$, by its reverse complement $\hat{\mathbf{b}}$.

The couple $(j, \ell)$ parametrizes the effect of an inversions/transpositions, which we denote by $g_{(j, \ell)}: \mathcal{A}^{N} \rightarrow \mathcal{A}^{N}$. Its action has interesting properties: $g_{(j, \ell)}$ is an involution for every $(j, \ell)$ and the total number of $C$ and $G$ (or, equivalently, of $A$ and $T)$ is invariant under $g: C G\left(\mathbf{s}_{t}\right)=C G\left(\mathbf{s}_{0}\right) \quad \forall t$. This implies that the dynamics is restricted to the invariant subspace of sequences with constant $\mathrm{CG}$ content $\mathcal{B}^{N}\left[C G\left(\mathbf{s}_{0}\right)\right]$.

Asymptotic equilibrium. The dynamics can be equivalently described as an ergodic Markov chain over the space of sequences $\mathcal{B}^{N}\left[C G\left(\mathbf{s}_{0}\right)\right]$. The fact that $g_{(j, \ell)}$ is an involution forces the transition matrix to be bi-stochastic and thus in the asymptotic equilibrium all sequences are equiprobable. This means that, for $t \rightarrow \infty$ and irrespective of the initial ancient DNA sequence, the evolution asymptotically leads to sequences that can be equivalently considered generated by an independent and identically distributed (iid) process with $p(G)=p(C)=C G\left(\mathbf{s}_{0}\right) / 2$ and $p(A)=p(T)=$ $\left(1-C G\left(\mathbf{s}_{0}\right)\right) / 2$. Therefore, the expected value of our indicators of symmetry and structure Eqs. (1) and (2) vanish asymptotically,

$$
\lim _{t \rightarrow \infty} I_{\text {str }}(\mathbf{s}(t))=\lim _{t \rightarrow \infty} I_{\text {sym }}(\mathbf{s}(t))=0,
$$

for any initial sequence $\mathbf{s}(0)$ [52]. This shows analytically that the TE dynamics asymptotically leads to Chargaff symmetric sequences, in agreement with previous claims [12]. However, this symmetric equilibrium is a (trivial) consequence of a full randomization. Therefore our results show also that the current explanation of the second Chargaff parity rule [12] is not satisfactory as it is not compatible with any structure, which is known to remain significant at distances of several thousands of nucleotides [30-38] (see also Fig. 1). Next we show that the same TE dynamics is rich enough by showing that symmetric sequences with nontrivial structure are generated pre-asymptotically as long-lived metastable states of TEs dynamics.

Symmetry and structure over time-three regimes. We now investigate symmetry and structure of the sequences $\mathbf{s}(t)$ by 
computing how our indicators $I_{\text {sym }}$ an $I_{\text {str }}$ depend on time $t$ (i.e., their values after $t$ applications of $g_{(j, \ell)}$ ). We show that Chargaff symmetry emerges much before equilibrium, together with a complex domainlike structure.

We first investigate structural properties of sequences after a finite number $t$ of iterations. We define a domain of $\mathbf{s}(t)$ as a subsequence of consecutive sites that have been involved in the same series of reverse-complement events. We then distinguish between domains of type $\Gamma$ and $\hat{\Gamma}$, depending on whether the number of transformations $g$ they were involved is even or odd, respectively. By definition, the starting sequence is composed by a single domain of type $\Gamma$. After one iteration it is split into three domains, two of type $\Gamma$ and one of type $\hat{\Gamma}$ of length $\ell_{1}$, corresponding to the subsequence involved in the first reverse-complement event. We now compute the average sizes $\left\langle\ell_{\Gamma}\right\rangle(t)$ and $\left\langle\ell_{\hat{\Gamma}}\right\rangle(t)$ of domains after $t$ iterations. Three regimes can be identified.

(i) For short times $t$, if $L \ll N$, the probability that the first few iterates all involve different subsequence is very high [53]. At each iterate, a subsequence of a domain of type $\Gamma$ of average size $\langle\ell\rangle=L$ is created, cutting a domain of type $\hat{\Gamma}$. Thus we have that in this regime:

$$
\left\langle\ell_{\hat{\Gamma}}\right\rangle(t)=L \text { and }\left\langle\ell_{\Gamma}\right\rangle(t)=N / t .
$$

This regime lasts until iterates start overlapping, which happens when $N / t \approx L$ and average domain sizes equalize $\left\langle\ell_{\hat{\Gamma}}\right\rangle(t)=\left\langle\ell_{\Gamma}\right\rangle(t)=L$. This regime is thus valid for $0<t \lesssim$ $t_{\text {metastable }}=N / L$.

(ii) For $t \gtrsim t_{\text {metastable }}=N / L$ a typical reverse-complement event will overlap with more than one domain. In this case all the domains that lie fully inside the subsequence involved in the reverse-complement event will change type (and position) without changing length; the domains at the border are instead split in two subdomains of different types. The randomness of this process guarantees that the already reached balance between the number and average length of the two domain types $\Gamma$ and $\hat{\Gamma}$ is not broken while their common average length decreases in time as

$$
\left\langle\ell_{\hat{\Gamma}}\right\rangle(t)=\left\langle\ell_{\Gamma}\right\rangle(t)=N / t .
$$

This second regime ends after a number of iterations $t \sim$ $t_{\text {equilibrium }}=N$ when equilibrium is reached.

(iii) For $t>t_{\text {equilibrium }}=N$ the average lengths stabilize at the stationary value,

$$
\left\langle\ell_{\hat{\Gamma}}\right\rangle(t)=\left\langle\ell_{\Gamma}\right\rangle(t)=1,
$$

and the sequence can be thought as a realization of the asymptotic equilibrium discussed above.

We now explain how structure $I_{\text {str }}(\mathbf{s}(t))$ and symmetry $I_{\mathrm{sym}}(\mathbf{s}(t))$ depend on the domain sizes $\left\langle\ell_{\Gamma}\right\rangle$ and $\left\langle\ell_{\hat{\Gamma}}\right\rangle$ and thus on the different regimes.

$I_{\text {str }}(\mathbf{s})$ : In order to identify the contribution of the dynamics in generating complex structural features, we consider an initial $\mathbf{s}(0)$ generated by an iid process [no structure, $I_{\text {str }}(0)=0$ ]. With this choice, a value $I_{\text {str }} \neq 0$ signals the construction, under the action of the dynamics, of different domain types. In particular, at $t_{\text {metastable }}$ and for $L \gg 1$, the total variance $\sigma_{\tau}^{2}$ can be estimated, using the law of total variance, as the sum of two components: one that measures variability of the mean of returns between domain types and the other measuring

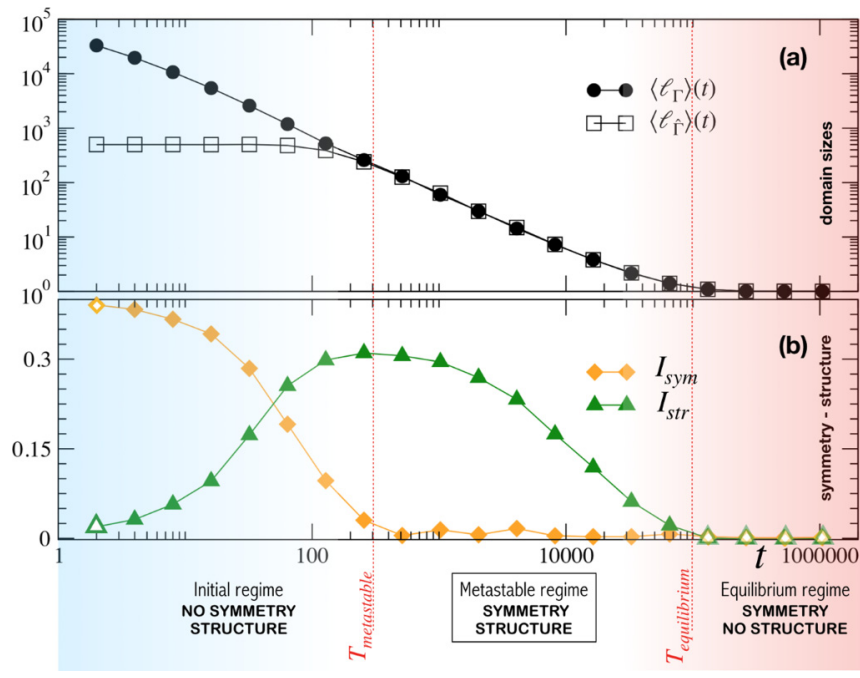

FIG. 2. Temporal evolution of symmetry and structure in the model. (a) Numerical evaluation of the average sizes of domains of the two types $\left\langle\ell_{\Gamma}\right\rangle(t)$ and $\left\langle\ell_{\hat{\Gamma}}\right\rangle(t)$ as a function of the number of iterates $t$ of TE's dynamics. (b) Numerical evaluation of the symmetry and structural properties of the sequence generated by the dynamics and quantified by the indicators $I_{\mathrm{str}}(t)$ and $I_{\text {sym }}(t)$. The filled symbols in $I_{\text {str }}$ indicate that these values are statistically different from a random sequence $[p$ value $<0.01$; equivalent results are obtained using as an alternative definition of $I_{\text {str }}$ the Jensen-Shannon divergence between the $P(\tau)$ obtained in the model and in random sequences]. The sequences $\mathbf{s}(t)$ have length $N=10^{5}$ and the size of reverse-complement events is $L=500$, thus leading to time scales $t_{\text {metastable }}=10^{2}$ and $t_{\text {equilibrium }}=10^{5}$. The starting sequence is fully random with $f_{A}=0.1, f_{C}=0.2, f_{G}=0.3, f_{T}=0.4$.

variability of returns within each type. Accordingly $I_{\text {str }}(t)$ grows from 0 to the value $I_{\text {str }}\left(t_{\text {metastable }}\right)>0$ at the end of the first regime. In the second regime the domain sizes decay and $I_{\mathrm{str}}(t)$ decreases to zero at equilibrium (at $t_{\text {equilibrium }}$ ). In terms of regimes we thus expect (i) $I_{\text {str }}$ grows; (ii) $I_{\text {str }}$ decays; (iii) $I_{\text {str }}=0$.

$I_{\mathrm{sym}}(\mathbf{s})$ : Each domain of type $\Gamma$ is a subsequence of the ancient sequence $\mathbf{s}(0)$. If average size of such domains at time $t$ is large enough, the frequencies of each nucleotide are approximately the same as their frequency in $\mathbf{s}(0)$; similarly for $\hat{\Gamma}$ and $\hat{\mathbf{s}}(0)$. No constraints are imposed to the symmetry of the ancient genome. In particular, if the original sequence is not Chargaff symmetric $I_{\mathrm{sym}}(\mathbf{s}(0))>0$ then the symmetry re-

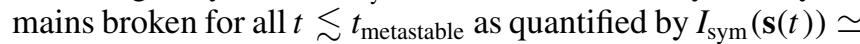
$\frac{t}{N}\left|\left\langle\ell_{\Gamma}\right\rangle(t)-\left\langle\ell_{\hat{\Gamma}}\right\rangle(t)\right| I_{\mathrm{sym}}(\mathbf{s}(0))$. In terms of regimes we thus expect (i) $I_{\mathrm{sym}}>0$; (ii) $I_{\mathrm{sym}}=0$; (iii) $I_{\mathrm{sym}}=0$.

Altogether, the estimations and calculations above lead to the following predictions for the presence of symmetry and structure as a function of time $t$ (regimes i-iii).

(i) $0 \leqslant t \leqslant t_{\text {metastable }}=N / L$ :

Structure $I_{\mathrm{str}}>0$ but no symmetry $I_{\mathrm{sym}}>0$.

(ii) $t_{\text {metastable }}=N / L \leqslant t \leqslant t_{\text {equilibrium }}=N$ :

Structure $I_{\mathrm{str}}>0$ and symmetry $I_{\mathrm{sym}}=0$.

(iii) $t_{\text {equilibrium }}=N<t$ :

Symmetry $I_{\text {sym }}=0$ but no structure $I_{\text {str }}=0$.

In Fig. 2 we confirm these predictions in a numerical simulation. 
The metastable regime. The crucial feature of the TE dynamics discussed above is that in regime (ii) both nontrivial structure and symmetry coexist in the generated sequences. The time (measured in number of iterations) for which this regime is valid is orders of magnitude larger than that of the first regime, as the ratio $t_{\text {equilibrium }} / t_{\text {metastable }}=L$ corresponds to the average size of transposable elements (for example, $L \simeq 10^{2}$ in Homo sapiens [46]). We thus denote such longlived regime as metastable and we expect it to be generically observed, even though it does not correspond to the stable equilibrium of our model.

The DNA sequences in the metastable regime are characterized by a symmetric domainlike structure. Domain models have been already introduced in literature to reproduce the complex structure generically observed in extant DNAs [11,40-46]. In particular if the distribution of domain sizes has a fat tail, this will lead to a long-range correlated sequence [11], signaled by a slow decay of $P(\tau)$. The novelty of our approach is twofold: First, the domainlike structure in the metastable regime is an emergent property of the TE dynamics (it is not imposed a priori); secondly, such complex structure is intertwined with symmetry, that itself is an output of the dynamics. In particular, we have shown that sequences in the metastable regime are not only Chargaff symmetric $\left(I_{\text {sym }}=0\right)$, they reproduce the hierarchical relation between symmetry and structure that is a distinctive feature of extant genomes (see Fig. 3).

Different organisms. In Fig. 4 we report $I_{\text {sym }}$ and $I_{\text {str }}$ computed for genomes of different families, together with the values obtained from our dynamics. It shows that symmetry and structure coexist in most cases. The sequences from animals show enhanced structure while the cases of archaea and bacteria show a moderate signature of structure, in agreement with the temporal behavior of our model (i.e., associating $t$ with the age of the genomes). Note that symmetry and structure properties are both statistical observations we made on the full DNA sequence. Any evolutionary constraint that pertains a small percentage of an organism genome does not affect these statistical observations in a sensible way. As an example, the protein-coding regions of Homo sapiens account for $1.5 \%$ of the full sequence. On the other hand, care should be taken when dealing with many different organisms: Extensions of the model incorporating additional aspects of DNA evolution will be required for a quantitative comparison with the empirical data.

Conclusion. We have shown how a model that captures the action of transposable elements (TEs) is able to reproduce the intricate relation between symmetry and structure present in DNA sequences. We find that symmetry and structure change differently at different time scales (i.e., for different number of actions of TEs). For a large (pre-asymptotic) time interval, the sequences obtained in our model show the same nontrivial structures and a hierarchy of symmetries (including Chargaff) as in actual DNA sequences [see Figs. 1(b) and 3(b)]. Our mathematical model is extremely simplified and includes the essential elements to explain the onset of symmetry and structure. In particular, it mimics only a simple action of TEs (reverse-complement), ignoring the fact that TEs are classified in different families, have different properties, and act according to different mechanisms [47-49]. We expect that
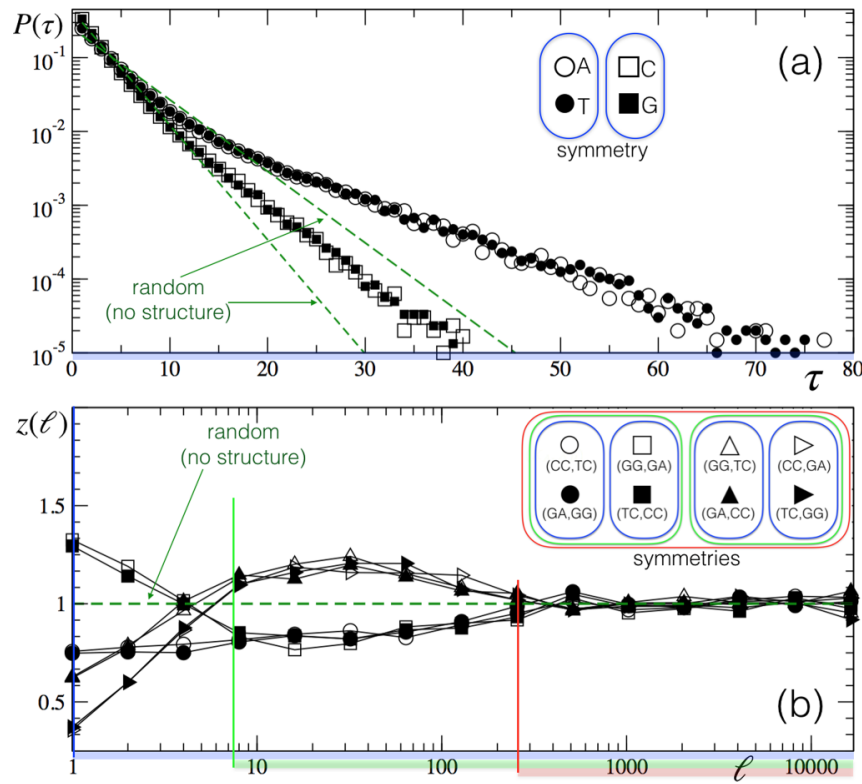

FIG. 3. Symmetry and structure in the metastable regime. Same observables as in Fig. 1 are computed for a sequence in the metastable regime of our dynamics. Data show that this regime is characterized by a similar co-occurrence of symmetry and structure as in extant genomes. Results in (a) are for a sequence of length $N=5 \times 10^{6}$ initialized as in Fig. 2 and evolved using our model with TE sizes $\ell$ all equals to $L=5000$ until $t=2048 \gtrsim t_{\text {metastable }}=1000$. Results in (b) are for a sequence of length $N=10^{5}$ initialized as in the artificial sequence reported in Ref. [39] and evolved using our dynamic model with fixed $L=500$ until $t=256 \gtrsim t_{\text {metastable }}=200$. The more generic initial sequence in (b) (i.e., Markov chain instead of fully random) allows us to distinguish between the different types of scale-dependent symmetries generated by the dynamics.

incorporating more details of the TE dynamics in our model will refine our understanding of their role in shaping statistical properties of DNA sequences, in particular in an evolutionary viewpoint that would lead to refinements in the data-model comparison presented in Fig. 4.

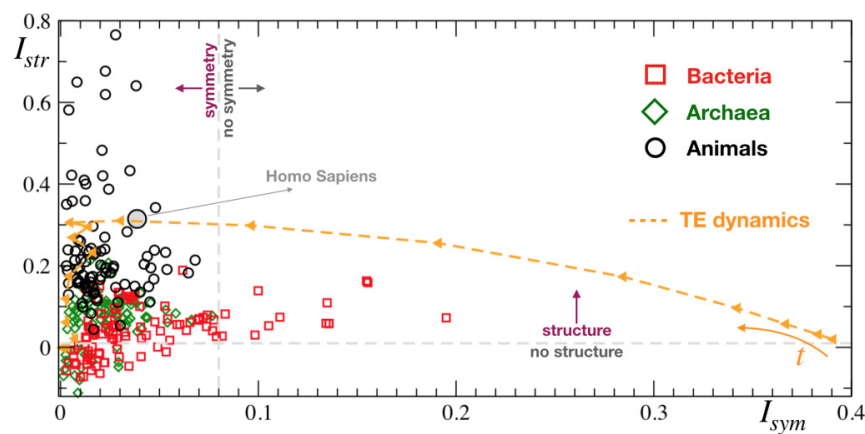

FIG. 4. Structure and symmetry in different organisms. Values of $I_{\text {sym }}$ vs $I_{\text {str }}$ for different genomes belonging to the families archaea, bacteria, and animals [50]. Superimposed are the values of the sequences evolved via our model (starting in $\left(I_{\text {sym }}, I_{\text {str }}\right)=(0.39,0)$ and evolving to $(0,0)$; parameters as in Fig. 2(a). 
[1] B. McClintock, The origin and behavior of mutable loci in maize, Proc. Natl. Acad. Sci. USA 36, 344 (1950).

[2] C. Feschotte and E. J. Pritham, DNA Transposons and the Evolution of Eukaryotic Genomes, Annu. Rev. Genet 41, 331 (2007).

[3] N. Kleckner, Transposable elements in prokaryotes, Annu. Rev. Gen. 15, 341 (1981).

[4] G. Bourque, K. H. Burns, M. Gehring, V. Gorbunova, A. Seluanov, M. Hammell, M. Imbeault, Z. Izsvák, H. L. Levin, T. S. Macfarlan, D. L. Mager, and C. Feschotte, Ten things you should know about transposable elements, Genome Biology 19, 199 (2018).

[5] N. V. Fedoroff, Transposable elements, epigenetics and genome evolution, Science 338, 758 (2012).

[6] D. Holste, I. Grosse, S. Beirer, P. Schieg, and H. Herzel, Repeats and correlations in human DNA sequences, Phys. Rev. E 67, 061913 (2003).

[7] M. Sheinman, A. Ramisch, F. Massip, and P. F. Arndt, Evolutionary dynamics of selfish DNA explains the abundance distribution of genomic subsequences, Sci. Rep. 6, 30851 (2016).

[8] F. Massip and P. F. Arndt, Neutral Evolution of Duplicated DNA: An Evolutionary Stick-Breaking Process Causes ScaleInvariant Behavior, Phys. Rev. Lett. 110, 148101 (2013).

[9] P. W. Messer, P. F. Arndt, and M. Lässig, Solvable Sequence Evolution Models and Genomic Correlations, Phys. Rev. Lett. 94, 138103 (2005).

[10] G. Attard, A. Hurworth, and J. Jack, Language-like features in DNA: transposable element footprints in the genome, Europhys. Lett. 36, 391 (1996).

[11] S. V. Buldyrev, A. L. Goldberger, S. Havlin, C. K. Peng, M. Simons, and H. E. Stanley, Generalized levy walk model for DNA nucleotide sequences, Phys. Rev. E 47, 4514 (1993).

[12] G. Albrecht-Buehler, Asymptotically increasing compliance of genomes with Chargaff's second parity rules through inversions and inverted transpositions, Proc. Natl. Acad. Sci. USA 103, 17828 (2006).

[13] R. Rudner, J. D. Karkas, and E. Chargaff, Separation of B. subtilis DNA into complementary strands I. Biological properties, II. Template functions and composition as determined, III Direct analysis, Proc. Natl. Acad. Sci. USA 60, 630; 915 (1968).

[14] A. C. Rogerson, There appear to be conserved constraints on the distribution of nucleotide sequences in cellular genomes, J. Mol. Evol. 32, 24 (1991).

[15] D. Mitchell and R. Bridge, A test of Chargaff's second rule, Biochem. Biophys. Res. Commun. 340, 90 (2006).

[16] C. Nikolaou and Y. Almirantis, Deviations from Chargaff's second parity rule in organellar DNA Insights into the evolution of organellar genomes, Gene 381, 34 (2006).

[17] D. Qi and A. J. Cuticchia, Compositional symmetries in complete genomes, Bioinformatics 17, 557 (2001).

[18] J. W. Fickett and D. C. Torney, Wolf DR Base compositional structure of genomes, Genomics 13, 1056 (1992).

[19] V. V. Prabhu, Symmetry observations in long nucleotide sequences, Nucleic Acids Res. 21, 2797 (1993).

[20] S. J. Bell and D. R. Forsdyke, Accounting units in DNA, J. Theor. Biol. 197, 51 (1999).

[21] P. F. Baisnée, S. Hampson, and P. Baldi, Why are complementary DNA strands symmetric?, Bioinformatics 18, 1021 (2002).
[22] S.-G. Kong, W.-L. Fan, H.-D. Chen, Z.-T. Hsu, N. Zhou, Bo Zheng, and H.-C. Lee, Inverse symmetry in complete genomes and whole-genome inverse duplication, PLOS one $\mathbf{4}$, e7553 (2009).

[23] V1 Afreixo, C. A. Bastos, S. P. Garcia, J. M. Rodrigues, A. J. Pinho, and P. J. Ferreira, The breakdown of the word symmetry in the human genome, J. Theor. Biol. 335, 153 (2013).

[24] S. J. Bell and D. R. Forsdyke, Deviations from chargaff's second parity rule correlate with direction of transcription, $\mathrm{J}$. Theor. Biol. 197, 63 (1999).

[25] J. R. Lobry and C. Lobry, Evolution of DNA base composition under no-strand-bias condition when the substitution rates are not constant, Mol. Biol. Evol. 16, 719 (1999).

[26] S. H. Zhang and Y. Z. Huang, Limited contribution of stemloop potential to symmetry of single-stranded genomic DNA, Bioinformatics 26, 478 (2010).

[27] A. Hart, S. Martínez, and F. A. Olmos, Gibbs approach to chargaff's second parity rule, J. Stat. Phys. 146, 408 (2012).

[28] L. A. Coons, A. B. Burkholder, S. C. Hewitt, D. P. McDonnell, and K. S. Korach, Decoding the inversion symmetry underlying transcription factor DNA-binding specificity and functionality in the genome, iScience 15, 552 (2019).

[29] P. Fariselli, C. Taccioli, L. Pagani, and A. Maritan, DNA sequence symmetries from randomness: The origin of the Chargaff's second parity rule, Briefings in Bioinformatics bbaa041 (2020).

[30] C. K. Peng, S. V. Buldyrev, A. L. Goldberger, S. Havlin, F. Sciortino, M. Simons, and H. E. Stanley, Long-range correlation in nucleotide sequences, Nature (London) 356, 168 (1992).

[31] W. Li and K. Kaneko, Long-range correlation and partial $1 / f^{\alpha}$ spectrum in a noncoding DNA sequence, Europhys. Lett. 17, 655 (1992).

[32] R. Voss, Evolution of Long-Range Fractal Correlations and $1 / f$ Noise in DNA Base Sequences, Phys. Rev. Lett. 68, 3805 (1992).

[33] I. Amato, DNA shows unexplained patterns writ large, Science 257, 747 (1992).

[34] P. Yam, Noisy nucleotides: DNA sequences show fractal correlations, Sci. Am. 267, 23-24 (1992).

[35] W. Li, T. G. Marr, and K. Kaneko, Understanding long-range correlations in DNA sequences, Physica D 75, 392 (1994).

[36] B. Audit, C. Thermes, C. Vaillant, J. d'Aubenton-Carafa, J. F. Muzy, and A. Arneodo, Long-Range Correlations in Genomic DNA: A Signature of the Nucleosomal Structure, Phys. Rev. Lett. 86, 2471 (2001).

[37] K. M. Frahm and D. L. Shepelyansky, Poincaré recurrences of DNA sequences, Phys. Rev. E 85, 016214 (2012).

[38] A. Colliva, R. Pellegrini, A. Testori, and M. Caselle, Isingmodel description of long-range correlations in DNA sequences, Phys. Rev. E 91, 052703 (2015).

[39] G. Cristadoro, M. Degli Esposti, and E. G. Altmann, The common origin of symmetry and structure in genetic sequences, Sci. Rep. 8, 15817 (2018).

[40] S. Nee, Uncorrelated DNA walks, Nature (London) 357, 450 (1992).

[41] S. Karlin and V. Brendel, Patchiness and correlations in DNA sequences, Science 259, 677 (1993).

[42] C. K. Peng, S. V. Buldyrev, S. Havlin, M. Simons, H.E. Stanley, and A. L. Goldberger, Mosaic organization of DNA nucleotides, Phys. Rev. E 49, 1685 (1994). 
[43] P. Bernaola-Galván, R. Román-Roldán, and J. L. Oliver, Compositional segmentation and long-range fractal correlations in DNA sequences, Phys. Rev. E 53, 5181 (1996).

[44] G. Bernardi, B. Olofsson, J. Filipski, M. Zerial, J. Salinas, G. Cuny, M. Meunier-Rotival, and F. Rodier, The mosaic genome of warm-blooded vertebrates, Science 228, 953 (1985).

[45] K. Rajeev, J. Azad, R. Subba, Li Wentian, and R. Ramakrishna, Simplifying the mosaic description of DNA sequences, Phys. Rev. E 66, 031913 (2002).

[46] P. Carpena, P. Bernaola-Galván, A. V. Coronado, M. Hackenberg, and J. L. Oliver, Identifying characteristic scales in the human genome, Phys. Rev. E 75, 032903 (2007).

[47] J. Jurka and T. Smith, A fundamental division in the Alu family of repeated sequences, Proc. Natl. Acad. Sci. USA 85, 4775 (1988).

[48] M. Munóz-López and J. L. García-Pérez, DNA transposons: nature and applications in genomics, Current Genomics 11, 115 (2010).

[49] J. Jurka, W. Bao, and K. K. Kojima, Families of transposable elements, population structure and the origin of species, Biology Direct 6, 44 (2011).
[50] See Supplemental Material at http://link.aps.org/supplemental/ 10.1103/PhysRevResearch.2.033477 for details on the data set used.

[51] More precisely, the pairs $(j, \ell)$ are drawn, independently from previous iterations, from a joint distribution $\rho(j, \ell)$ chosen such that its marginal $\phi(\ell)$ has support contained in $[0, N]$, finite average $L$, and the conditional distribution of positions $\psi(j \mid \ell)$ is uniform in [1,N- $N+1]$. We consider distributions $\phi(\ell)$ that guarantee ergodicity of the Markov Chain. We expect ergodicity to be generically valid; e.g., it suffices to have non-zero probability for the identity transformation (i.e. $\phi(0) \neq 0$ ) and for single-nucleotide complementing $(\psi(1) \neq 0)$.

[52] We can intuitively understand this result by noting that each action of the transposon effectively creates two cuts in the sequence and moves them by a distance $L$ on average. Since cuts can happen at any location, this process eventually mixes complementary basis at different positions and breaks any correlations originally present in $\mathbf{s}(0)$.

[53] Quantitatively, if we drop $t \leqslant N / L$ points uniformly at random on an interval of length $N$, they will be separated by a distance at least $L$ with probability $(1-(t-1) L / N)^{t}$. 